\title{
Cerium oxide nanoparticles in cancer
}

This article was published in the following Dove Press journal:

OncoTargets and Therapy

27 May 2014

Number of times this article has been viewed

Ying Gao'

Kan Chen ${ }^{2, *}$

Jin-lu Ma',*

Fei $\mathrm{Gao}^{3}$

'Department of Radiotherapy

Oncology, First Affiliated Hospital

of Medical College of Xi'an Jiaotong

University, Xi'an, People's Republic

of China; ${ }^{2}$ School of Life Sciences,

Zhejiang Sci-Tech University,

Hangzhou, People's Republic of China;

${ }^{3}$ Department of Neurology, First

Affiliated Hospital of Xi'an Medical

University, Xi'an, People's Republic of

China

*These authors contributed equally to this work
Correspondence: Ying Gao

Department of Radiotherapy Oncology, First Affiliated Hospital of Medical College of Xi'an Jiaotong University, 277 West Yanta Road, Xi'an, Shaanxi 7I 006 I, People's Republic of China

Tel +862985324019

Email togaoying@।63.com
Abstract: With the development of many nanomedicines designed for tumor therapy, the diverse abilities of cerium oxide nanoparticles (CONPs) have encouraged researchers to pursue CONPs as a therapeutic agent to treat cancer. Research data have shown CONPs to be toxic to cancer cells, to inhibit invasion, and to sensitize cancer cells to radiation therapy and chemotherapy. CONPs also display minimal toxicity to normal tissues and provide protection from various forms of reactive oxygen species generation. Differential cytotoxicity is important for anticancer drugs to distinguish effectively between tumor cells and normal cells. The antioxidant capabilities of CONPs, which enable cancer therapy protection, have also resulted in the exploration of these particles as a potential anticancer treatment. Taken together, CONPs might be a potential nanomedicine for cancer therapy and this review highlights the current research into CONPs as a novel therapeutic for the treatment of cancer.

Keywords: cerium oxide nanoparticles, cancer treatment, radioprotection, radiosensitization

\section{Introduction}

Nanotechnology has become a main focus of biomedical research in recent years. Nanomaterials show many useful and unique properties and can be applied in the areas of medicine, biology, and life science research. ${ }^{1-6}$ Nanoparticle applications include drug delivery systems, luminescent biomarkers, and tissue engineering, among others. ${ }^{7}$ Many nanomedicines have been designed for tumor therapy with the rapid development of nanoscience and nanotechnology. Nanomedicines are emerging as one of these new treatment options ${ }^{8,9}$ since it is necessary to explore novel drugs ${ }^{10,11}$ when the conventional therapies, including surgical interventions, radiation, and cytotoxic chemotherapies, are ineffective in curing cancer. ${ }^{8-12}$ In particular, cerium oxide nanoparticles (CONPs), which consist of a cerium core surrounded by an oxygen lattice, have shown promise in a number of applications. The tissue or cell environmental conditions appear to play an important role in the determination of activity, as CONPs also possess direct oxidant behavior despite the fact that CONPs have been shown to display a number of antioxidant behaviors. ${ }^{13-17}$ To date, $\mathrm{pH}$ is one of the few factors shown to drive whether CONPs act as oxidants or antioxidants. ${ }^{18,19}$

The reactive oxygen species (ROS) can drive both the initial development and progression of cancer, as well as downregulate antioxidant enzymes that normally combat radical production. ${ }^{20}$ In normal, healthy cells, the cellular levels of ROS are tightly controlled. ${ }^{21}$ The ability to modulate the redox status of cells has applications in diseases in which ROS levels have become deregulated or are altered by treatment. Some studies have shown CONPs to possess innate cytotoxicity to cancer cells, 
anti-invasive properties, and the ability to sensitize cancer cells to radiation-induced cell death, while protecting the surrounding normal tissues. ${ }^{18-21}$ Therefore, CONPs have extensive potential as a therapeutic agent for the treatment of cancer, as well as other diseases in which ROS have been implicated. ${ }^{22}$ Potential applications and prospects of CONPs in cancer are summarized herein.

\section{Antitumor aspects}

Cerium oxide is prepared by the supercritical synthesis method. There are two main ways of making the particles chemical or flame spray pyrolysis. ${ }^{23}$ It has been reported that many nanomedicines with chemical modifications can kill tumor cells by increasing ROS level in tumor cells or by targeting the nucleus or other organ cells. ${ }^{12,24-56}$ As for CONPs, a study has demonstrated their toxicity to cancer cells and inhibition of the metastasis and polymer-coated CONPs to manipulate tumor-stroma interactions to the detriment of tumor progression and invasion. ${ }^{28}$ Another study found that CONPs caused cytochrome c release and activated caspase- 3 and caspase-9, which demonstrates that CONPs induced the apoptosis of tumor cells by initiating a mitochondrion-mediated apoptosis signaling pathway without chemical modification, specifically targeting the mitochondria. ${ }^{12}$

Polymer coating of CONPs increases aqueous solubility, ${ }^{18}$ but it does not appear to impact CONP redox activities. ${ }^{13,28}$ Myofibroblasts largely mediate epithelial/ stromal signaling. They play a key role in the expression of extracellular matrix components, including alpha-smooth muscle actin and collagen, to facilitate tumor invasion and angiogenesis. ${ }^{29}$ Data show that CONPs possess the ability to modulate myofibroblast formation with the transition from fibroblast to myofibroblast driven by TGF$\beta 1$-induced ROS-dependent expression of smooth muscle actin. Pretreatment with CONPs mitigated both the corresponding myofibroblast transition and TGF- $\beta 1$-induced alpha-smooth muscle actin expression in fibroblasts. ${ }^{7,28}$ As some myofibroblasts localize to the invasion front of tumors, CONP treatment diminished the ability of myofibroblasts to induce invasion by squamous tumor cells. ${ }^{28}$ In addition, CONPs are also able to decrease the intrinsic ability of cultured squamous tumor cells to invade, even in the absence of any myofibroblast stimulation. To sum up, these results demonstrate the direct negative effects of CONPs on cancer cells, as well as their ability to modulate the tumor environment and indirectly inhibit tumor cell invasion. These data also suggest that CONPs as a new type of antitumor nanomedicine can be applied to the treatment of cancer ultimately. ${ }^{28}$

\section{Radioprotection and radiosensitization}

In addition to surgery and chemotherapy, radiation therapy (RT) remains a mainstay in the treatment of cancer. ${ }^{29}$ However, the side effects of RT remain the most challenging issue for cancer treatment. Many harmful side effects are associated with RT, including nausea, fatigue, and dermatitis, ${ }^{30,31}$ but few radiation adjuvants are available to mitigate these painful outcomes. At present, the clinically available radioprotectant drugs are not ideal. These include amifostine, which remains the radioprotectant, and which is itself associated with nausea and hypotension. ${ }^{32,33}$ Thus, it is necessary to explore novel drugs. The ability of CONPs to modulate ROS has led to their exploration for the improvement of RT. The dual capabilities of CONPs to act as an oxidant in cancer cells, yet antioxidant in normal cells, supports the role of CONPs as an adjuvant for RT that could significantly impact patient quality of life. Several publications have shown that treatment with CONPs prior to RT exposure decreases the RT-induced cell damage and death in normal tissues in line with the protection from other methods of inducing oxidative stress. ${ }^{34-37}$ One study suggests that $\mathrm{CeO}_{2}$ may be radioprotective for salivary production and reduce grade III dermatitis and skin hyperpigmentation incidence ${ }^{37} \mathrm{CeO}_{2}$ as radioprotectant may be a feasible concept during radiotherapy. ${ }^{37} \mathrm{CONP}$ radical scavenging was found to inhibit the resulting caspase- 3 activation in irradiated colonic crypt tissue, as well as caspase- 3 and -7 activation mechanistically in irradiated lung fibroblasts in culture..$^{34,35}$ In addition, CONPs increased super oxide dismutase 2 (SOD2) expression up to twofold in a dose-dependent manner in normal human colon cells in vitro, while increasing SOD2 expression by $40 \%$ in colonic crypt cells from mice treated with CONPs. ${ }^{34}$ The aquatic environment and the size of CONPs are important. $\mathrm{pH}$, and phosphate ligands might play important roles in controlling the solubility of $\mathrm{CeO}_{2} \cdot{ }^{38} \mathrm{pH}$ is one of the few factors shown to drive whether CONPs act as oxidants or antioxidants. ${ }^{18,19}$ The antioxidant capabilities of CONPs have also resulted in the exploration of these particles as a potential treatment for other disorders characterized by ROS accumulation, which enables radiation protection. Thus, $\mathrm{pH}$ is an important factor for CONPs' appearance as nontoxic in normal cells because of a different $\mathrm{pH}$ in normal cells compared to tumor cells. ${ }^{19}$ In addition, the size and size distribution of the major components of the CONP solution are the most dominant factors for determining the dispersibility of 
CONPs in solution. ${ }^{39}$ Taken together, CONPs display minimal toxicity to normal tissues and provide protection from various forms of ROS generation. ${ }^{19}$ They may protect normal cells indirectly by priming cells to respond to ROS insult or directly by scavenging cellular ROS.

In contrast, CONPs have been found to be toxic to cancer cells, inhibit invasion, and sensitize cancer cells to RT. Pretreatment with CONPs has been shown to enhance the ability of RT to induce cell death in cancer cells with acidic $\mathrm{pH}$. Some studies showed that CONP treatment prior to RT markedly potentiated the cancer cell apoptosis, both in culture and in tumors, and the inhibition of the pancreatic tumor growth without harming the normal tissues or host mice., ${ }^{7,19}$ The results identify CONPs as potentially novel RT sensitizers as well as protectants for improving pancreatic cancer treatment. It has been suggested that CONPs in cancer cells are only capable of catalyzing the conversion of highly unstable superoxide to far more stable $\mathrm{H}_{2} \mathrm{O}_{2}$, as acidic $\mathrm{pH}$ has been shown to inhibit the catalase activity of CONPs. ${ }^{7,28}$ CONPs actually enhance the toxicity of RT in cancer cells by encouraging the accumulation and stability of ROS in the cell without the ability to act as a catalase mimetic and remove $\mathrm{H}_{2} \mathrm{O}_{2}$. These effects resulted in the radiosensitization of pancreatic cancer, significantly decreasing cell viability in vitro. ${ }^{19}$ In a pancreatic tumor-bearing mouse model that received the combination therapy of CONPs prior to RT, significant decreases in tumor weight and volume occurred with an increase in the number of apoptotic cells in the tumors. ${ }^{19}$

In all, these data demonstrate that CONPs modulate ROS in cancer cells such that, not only are there direct toxic effects, but the therapeutic properties of CONPs potentially extend to radioprotection and radiosensitization of cancer therapies.

\section{CONP prospects in cancer treatment}

CONPs are widely reported to be noncytotoxic and modulate intracellular ROS. The level of nanoceria surface functionalization with heparin determines the intracellular localization and ROS scavenging ability of these particles. Heparin-nanoceria was effective in reducing endothelial cell proliferation, indicating that they may have application in the control of angiogenesis in cancer in the future. ${ }^{40}$ It has been shown that CONPs have a unique property of inducing angiogenesis, which is critical for many physiological and pathophysiological processes and promotes the formation of new blood vessels from existing blood vessels. ${ }^{40}$ In particular, CONPs trigger angiogenesis by modulating the intracellular oxygen environment and stabilizing hypoxia, inducing factor $1 \alpha$ endogenously. Additionally, correlations between angiogenesis induction and CONP physicochemical properties, including surface $\mathrm{Ce}^{3+} / \mathrm{Ce}^{4+}$ ratio, surface charge, size, and shape, have also been explored. Increased $\mathrm{Ce}^{3+} / \mathrm{Ce}^{4+}$ ratio and high surface area make CONPs more catalytically active toward regulating intracellular oxygen, which in turn leads to more robust induction of angiogenesis. Atomistic simulation was also used to reveal that the surface reactivity of CONPs and facile oxygen transport promotes angiogenesis. $^{41}$

Furthermore, various nanoparticle-based approaches to overcome efflux-mediated resistance have been investigated, ${ }^{42}$ such as the use of formulation excipients that inhibit transporter activity and co-delivery of the anticancer drug with a specific inhibitor of transporter function or expression. However, the effectiveness of nanoparticles can be diminished by poor transport in the tumor tissue. Hence, to overcome that, adjunct therapies that improve the intratumoral distribution of nanoparticles may be vital to the successful application of nanotechnology. Coadministration of the chemotherapeutic and efflux inhibitor in nanoparticles may allow temporal colocalization of some unfavorable molecules, limiting their nonspecific distribution and hence their toxicities. ${ }^{42}$ In addition, another study has shown that some of the excipients used in the construction of nanoparticles are capable of inhibiting efflux transporters. ${ }^{43}$ Taken together, nanotechnology offers a promising approach for overcoming efflux pump-based drug resistance.

Curcumin has been used in the treatment of inflammatory disorders and cancer for many years. ${ }^{38,44-47}$ Curcumin may inhibit tumor growth via multiple mechanisms, including antitumor angiogenesis, suppression of proliferation, induction of apoptosis, and prevention of metastasis. ${ }^{48-53}$ There is some evidence suggesting that curcumin is an ideal chemosensitizer for chemotherapy and that it helps to protect patients from the side effects of treatment. ${ }^{54-58}$ Curcumin chemosensitizes because it is a highly effective scavenger of ROS and also inhibits the c-Jun $\mathrm{NH}_{2}-$ terminal kinase pathway. ${ }^{59}$ Both ROS and activation of the c-Jun $\mathrm{NH}_{2}$-terminal kinase pathway are crucial elements in the success of chemotherapy. ${ }^{56}$ However, the clinical applications of curcumin remain limited because of its short biological half-life, poor solubility resulting in poor absorption, and low bioavailability via the oral route. ${ }^{60-62}$ Recently, many novel chemotherapeutic formulations have been developed. These formulations contain chemotherapeutic agents inside the vehicle, resulting in 
better drug penetration into tumor tissue and less toxicity. Biodegradable polymeric nanoparticles are often used to achieve controlled release of drugs in advanced anticancer drug delivery systems. ${ }^{63-66}$ Further, some biodegradable polymer-derived drug delivery systems, such as nanoparticles delivering anticancer agents, are commercially available. ${ }^{67}$ Despite overcoming drug resistance to chemotherapy and the development of chemosensitizers from nanoparticles, there is still little research concerning CONP in this context. Future studies involving CONP application in chemotherapy are anticipated.

Some studies have shown that CONPs can selectively induce apoptosis and suppress the proliferation of tumor cells. Wang et al showed that nanoparticles can target specific organs, have a lower toxicity against the whole organism, and have good dissolubility in water. ${ }^{1}$ In their study, low concentrations of CONPs selectively killed tumor cells; the inability to clear CONPs might be one of the mechanisms that caused the CONPs to show selective cytotoxicity against tumor cells. ${ }^{1}$ Other results demonstrated that CONPs not only significantly delayed the growth of subcutaneous melanoma, but also increased the survival rate of tumor-bearing mice without damage to the organs. ${ }^{12}$ Importantly, the results also indicated that CONPs were rapidly cleared from the organs and that these particles exhibited little systemic toxicity. ${ }^{12}$ In addition, published data indicate that CONPs are toxic to bronchial epithelial lung fibroblasts in culture, but nontoxic to mammary epithelial cells, macrophages, immortalized keratinocytes, or immortalized pancreatic epithelial cells. ${ }^{7}$ The physiological $\mathrm{pH}$ in normal cells, to which CONPs are not toxic, enables canonical radical scavenging by CONPs. ${ }^{19,23,68,69}$ Therefore, CONPs introduced prior to ROS insult confer protection from the effects of oxidative stress in vitro and in vivo. ${ }^{70-73}$

On the other hand, CONPs are toxic to several types of human cancer cells. ${ }^{19,28,74}$ Cellular toxicity is attributed to the generation of ROS and the induction of oxidative stress, at least in part by the inherent oxidase activity of the nanoparticle core at acidic $\mathrm{pH}$ similar to that of cancer cells. ${ }^{13,19,28,69}$ CONPs can also produce ROS and initiate lipid peroxidation of the liposomal membrane, thereby regulating many signaling pathways and influencing the vital movements of cells. In particular, CONP treatment has been shown to induce glutathione oxidation, lipid peroxidation, and membrane damage in lung cancer cells. ${ }^{74}$ The generation of CONPs with a negative surface charge can induce preferential accumulation in acidic lysosomes within the cell, resulting in increased toxicity in cancer cells.

\section{Conclusion}

Differential cytotoxicity is important because one of the greatest challenges in chemotherapy is the inability of anticancer drugs to distinguish effectively between tumor cells and normal cells. Taken together, despite the fact that the number of potential applications for CONP-based therapies appears countless, given that ROS and oxidative stress linked to so many conditions, these results demonstrate that CONPs have selective cytotoxicity toward tumor cells, and indicate that CONPs might be a potential nanomedicine for cancer therapy and results pertaining to the potential application of CONPs for the treatment of numerous diseases are overwhelmingly positive thus far.

\section{Acknowledgments}

This study was supported by the National Natural Science Foundation of China (No 81301937) and by the International Cooperation Foundation of Shaanxi Province of China (No 2013KW-27-03).

\section{Disclosure}

The authors report no conflicts of interest in this work. This paper has not been published previously. This study will not be published elsewhere in the same form, in English or in any other language, without consent of the publisher.

\section{References}

1. Wang Y, Zi XY, Su J, et al. Cuprous oxide nanoparticles selectively induce apoptosis of tumor cells. Int $J$ Nanomedicine. 2012;7: 2641-2652.

2. Yang F, Tang Q, Zhong X, et al. Surface decoration by Spirulina polysaccharide enhances the cellular uptake and anticancer efficacy of selenium nanoparticles. Int J Nanomedicine. 2012;7:835-844.

3. Lee P, Zhang R, Li V, et al. Enhancement of anticancer efficacy using modified lipophilic nanoparticle drug encapsulation. Int $J$ Nanomedicine. 2012;7:731-737.

4. Murphy EA, Majeti BK, Barnes LA, et al. Nanoparticle-mediated drug delivery to tumor vasculature suppresses metastasis. Proc Natl Acad Sci U S A. 2008;105:9343-9348.

5. Siddiqui IA, Adhami VM, Chamcheu JC, Mukhtar H. Impact of nanotechnology in cancer: emphasis on nanochemoprevention. Int $J$ Nanomedicine. 2012;7:591-605.

6. Ghadiali JE, Cohen BE, Stevens MM. Protein kinase-actuated resonance energy transfer in quantum dot - peptide conjugates. ACS Nano. 2010;4:4915-4919.

7. Wason MS, Zhao J. Cerium oxide nanoparticles: potential applications for cancer and other diseases. Am J Transl Res. 2013;5:126-131.

8. Lee SK, Kim GS, Wu Y, et al. Nanowire substrate-based laser scanning cytometry for quantitation of circulating tumor cells. Nano Lett. 2012;12:2697-2704.

9. Dicheva BM, ten Hagen TL, Li L, et al. Cationic thermosensitive liposomes: a novel dual targeted heat-triggered drug delivery approach for endothelial and tumor cells. Nano Lett. 2013;13:2324-2331.

10. Kanapathipillai M, Mammoto A, Mammoto T, et al. Inhibition of mammary tumor growth using lysyl oxidase-targeting nanoparticles to modify extracellular matrix. Nano Lett. 2012;12:3213-3217. 
11. Zhao G, Rodriguez BL. Molecular targeting of liposomal nanoparticles to tumor microenvironment. Int J Nanomedicine. 2013;8:61-71.

12. Wang Y, Yang F, Zhang HX, et al. Cuprous oxide nanoparticles inhibit the growth and metastasis of melanoma by targeting mitochondria. Cell Death Dis. 2013;4:e783.

13. Asati A, Santra S, Kaittanis C, Nath S, Perez JM. Oxidase-like activity of polymer-coated cerium oxide nanoparticles. Angew Chem Int Ed Engl. 2009;48:2308-2312.

14. Korsvik C, Patil S, Seal S, Self WT. Superoxide dismutase mimetic properties exhibited by vacancy engineered ceria nanoparticles. Chem Commun (Camb). 2007;14:1056-1058.

15. Pirmohamed T, Dowding JM, Singh S, et al. Nanoceria exhibit redox state-dependent catalase mimetic activity. Chem Commun (Camb) 2010;46:2736-2738.

16. Dowding JM, Dosani T, Kumar A, Seal S, Self WT. Cerium oxide nanoparticles scavenge nitric oxide radical ( $\mathrm{NO}$ ). Chem Commun (Camb). 2012;48:4896-4898.

17. Xue Y, Luan QF, Yang D, Yao X, Zhou K. Direct evidence for hydroxyl radical scavenging activity of cerium oxide nanoparticles. JPhys Chem C Nanomater Interfaces. 2011;115:4433-4438.

18. Asati A, Santra S, Kaittanis C, Perez JM. Surface-charge-dependent cell localization and cytotoxicity of cerium oxide nanoparticles. $A C S$ Nano. 2010;4:5321-5331.

19. Wason MS, Colon J, Das S, et al. Sensitization of pancreatic cancer cells to radiation by cerium oxide nanoparticle-induced ROS production. Nanomedicine. 2013,9:558-569.

20. Waris G, Ahsan H. Reactive oxygen species: role in the development of cancer and various chronic conditions. $J$ Carcinog. 2006;5:14.

21. Ray PD, Huang BW, Tsuji Y. Reactive oxygen species (ROS) homeostasis and redox regulation in cellular signaling. Cell Signal. 2012;24:981-990.

22. Rzigalinski BA, Meehan K, Davis RM, Xu Y, Miles WC, Cohen CA. Radical nanomedicine. Nanomedicine (Lond). 2006;1:399-412.

23. Park EJ, Choi J, Park YK, Park K. Oxidative stress induced by cerium oxide nanoparticles in cultured BEAS-2B cells. Toxicology. 2008;245:90-100.

24. Dam DH, Lee JH, Sisco PN, et al. Direct observation of nanoparticlecancer cell nucleus interactions. ACS Nano. 2012;6:3318-3326.

25. Ma X, Zhang LH, Wang LR, et al. Single-walled carbon nanotubes alter cytochrome c electron transfer and modulate mitochondrial function. ACS Nano. 2012;6:10486-10496.

26. Huanga JG, Leshuk T, Gu FX. Emerging nanomaterials for targeting subcellular organelles. Nano Today. 2011;6:478-492.

27. Wang L, Liu Y, Li W, et al. Selective targeting of gold nanorods at the mitochondria of cancer cells: implications for cancer therapy. Nano Lett. 2011;11:772-780.

28. Alili L, Sack M, Karakoti AS, et al. Combined cytotoxic and antiinvasive properties of redox-active nanoparticles in tumor-stroma interactions. Biomaterials. 2011;32:2918-2929.

29. Desmoulière A, Guyot C, Gabbiani G. The stroma reaction myofibroblast: a key player in the control of tumor cell behavior. Int J Dev Biol. 2004;48:509-517.

30. Papakostidi A, Tolia M, Tsoukalas N. Quality assurance in Health Services: the paradigm of radiotherapy. J BUON. 2014;191:47-52.

31. Sveistrup J, af Rosenschöld PM, Deasy JO, et al. Improvement in toxicity in high risk prostate cancer patients treated with image-guided intensity-modulated radiotherapy compared to $3 \mathrm{D}$ conformal radiotherapy without daily image guidance. Radiat Oncol. 2014;9:44.

32. Citrin D, Cotrim AP, Hyodo F, Baum BJ, Krishna MC, Mitchell JB Radioprotectors and mitigators of radiation-induced normal tissue injury. Oncologist. 2010;15:360-371.

33. Kouvaris JR, Kouloulias VE, Vlahos LJ. Amifostine: the first selective-target and broad-spectrum radioprotector. Oncologist. 2007;12:738-747.

34. Colon J, Hsieh N, Ferguson A, et al. Cerium oxide nanoparticles protect gastrointestinal epithelium from radiation-induced damage by reduction of reactive oxygen species and upregulation of superoxide dismutase 2 . Nanomedicine. 2010;6:698-705.
35. Colon J, Herrera L, Smith J, et al. Protection from radiation-induced pneumonitis using cerium oxide nanoparticles. Nanomedicine. 2009;5: $225-231$.

36. Tarnuzzer RW, Colon J, Patil S, Seal S. Vacancy engineered ceria nanostructures for protection from radiation-induced cellular damage. Nano Lett. 2005;5:2573-2577.

37. Madero-Visbal RA, Alvarado BE, Colon JF, et al. Harnessing nanoparticles to improve toxicity after head and neck radiation. Nanomedicine. 2012;8:1223-1231.

38. Dahle JT, Livi K, Arai Y. Effects of $\mathrm{pH}$ and phosphate on $\mathrm{CeO} 2$ nanoparticle dissolution. Chemosphere. Epub March 11, 2014.

39. Arita T, Yoo J, Ueda Y, Adschiri T. Size and size distribution balance the dispersion of colloidal $\mathrm{CeO} 2$ nanoparticles in organic solvents. Nanoscale. 2010;2:689-693.

40. Das S, Singh S, Dowding JM, et al. The induction of angiogenesis by cerium oxide nanoparticles through the modulation of oxygen in intracellular environments. Biomaterials. 2012;33:7746-7755.

41. Lord MS, Tsoi B, Gunawan C, Teoh WY, Amal R, Whitelock JM. Anti-angiogenic activity of heparin functionalised cerium oxide nanoparticles. Biomaterials. 2013;34:8808-8818.

42. Patil Y, Sadhukha T, Ma L, Panyam J. Nanoparticle-mediated simultaneous and targeted delivery of paclitaxel and tariquidar overcomes tumor drug resistance. J Control Release. 2009;136:21-29.

43. Kabanov AV, Batrakova EV, Alakhov VY. Pluronic block copolymers for overcoming drug resistance in cancer. Adv Drug Deliv Rev. 2002;54:759-779.

44. Bharti AC, Donato N, Singh S, Aggarwal BB. Curcumin (diferuloylmethane) down-regulates the constitutive activation of nuclear factor-kappa B and IkappaBalpha kinase in human multiple myeloma cells, leading to suppression of proliferation and induction of apoptosis. Blood. 2003;101:1053-1062.

45. Jobin C, Bradham CA, Russo MP, et al. Curcumin blocks cytokinemediated NF-kappa B activation and proinflammatory gene expression by inhibiting inhibitory factor I-kappa B kinase activity. J Immunol. 1999;163:3474-3483.

46. Kunnumakkara AB, Diagaradjane P, Guha S, et al. Curcumin sensitizes human colorectal cancer xenografts in nude mice to gamma-radiation by targeting nuclear factor-kappaB-regulated gene products. Clin Cancer Res. 2008;14:2128-2136.

47. Anand P, Sundaram C, Jhurani S, Kunnumakkara AB, Aggarwal BB Curcumin and cancer: an "old-age" disease with an "age-old" solution. Cancer Lett. 2008;267:133-164.

48. Arbiser JL, Klauber N, Rohan R, et al. Curcumin is an in vivo inhibitor of angiogenesis. Mol Med. 1998;4:376-383.

49. Lin YG, Kunnumakkara AB, Nair A, et al. Curcumin inhibits tumor growth and angiogenesis in ovarian carcinoma by targeting the nuclear factor-kappaB pathway. Clin Cancer Res. 2007;13:3423-3430.

50. Dorai T, Cao YC, Dorai B, Buttyan R, Katz AE. Therapeutic potential of curcumin in human prostate cancer. III. Curcumin inhibits proliferation, induces apoptosis, and inhibits angiogenesis of $\mathrm{LNCaP}$ prostate cancer cells in vivo. Prostate. 2001;47:293-303.

51. Kunnumakkara AB, Anand P, Aggarwal BB. Curcumin inhibits proliferation, invasion, angiogenesis and metastasis of different cancers through interaction with multiple cell signaling proteins. Cancer Lett. 2008;269:199-225.

52. van't Land B, Blijlevens NM, Marteijn J, et al. Role of curcumin and the inhibition of NF-kappaB in the onset of chemotherapy-induced mucosal barrier injury. Leukemia. 2004;18:276-284.

53. Bhaumik S, Anjum R, Rangaraj N, Pardhasaradhi BV, Khar A. Curcumin mediated apoptosis in AK-5 tumor cells involves the production of reactive oxygen intermediates. FEBS Lett. 1999;456:311-314.

54. Duvoix A, Blasius R, Delhalle S, et al. Chemopreventive and therapeutic effects of curcumin. Cancer Lett. 2005;223:181-190.

55. Mitchell TM. Somasundaram, et al. Dietary curcumin inhibits chemotherapy-induced apoptosis in models of human breast cancer. Cancer Res. 2002;62:3868-3875.

56. Somasundaram S, Edmund NA, Moore DT, Small GW, Shi YY, Orlowski RZ. Dietary curcumin inhibits chemotherapy-induced apoptosis in models of human breast cancer. Cancer Res. 2002;62: 3868-3875. 
57. Du B, Jiang L, Xia Q, Zhong L. Synergistic inhibitory effects of curcumin and 5-fluorouracil on the growth of the human colon cancer cell line HT-29. Chemotherapy. 2006;52:23-28.

58. Patel BB, Sengupta R, Qazi S, et al. Curcumin enhances the effects of 5-fluorouracil and oxaliplatin in mediating growth inhibition of colon cancer cells by modulating EGFR and IGF-1R. Int J Cancer. 2008; 122:267-273.

59. Chen YR, Tan TH. Inhibition of the c-Jun N-terminal kinase (JNK) signaling pathway by curcumin. Oncogene. 1998;17:173-178.

60. Bisht S, Feldmann G, Soni S, et al. Polymeric nanoparticle-encapsulated curcumin ("nanocurcumin"): a novel strategy for human cancer therapy. J Nanobiotechnology. 2007;5:3.

61. Lao CD, Ruffin MT 4th, Normolle D, et al. Dose escalation of a curcuminoid formulation. BMC Complement Altern Med. 2006;6:10.

62. Bisht K, Wagner KH, Bulmer AC. Curcumin, resveratrol and flavonoids as anti-inflammatory, cyto- and DNA-protective dietary compounds. Toxicology. 2010;278:88-100.

63. Shutava TG, Balkundi SS, Vangala P, et al. Layer-by-layer-coated gelatin nanoparticles as a vehicle for delivery of natural polyphenols. ACS Nano. 2009;3:1877-1885.

64. Shieh YA, Yang SJ, Wei MF, Shieh MJ. Aptamer-based tumor targeted drug delivery for photodynamic therapy. ACS Nano. 2010;4: 1433-1442.

65. Wang T, He N. Preparation, characterization and applications of lowmolecular-weight alginate-oligochitosan nanocapsules. Nanoscale. 2010;2:230-239.
66. He N, Wang T, Jiang L, Wang D, Hu Y, Zhang L. Therapy for cerebral ischemic injury with erythropoietin-containing nanoparticles. J Nanosci Nanotechnol. 2010;10:5320-5323.

67. Wang X, Yang L, Chen ZG, Shin DM. Application of nanotechnology in cancer therapy and imaging. CA Cancer J Clin. 2008;58:97-110.

68. Hirst SM, Karakoti AS, Tyler RD, Sriranganathan N, Seal S, Reilly CM. Anti-inflammatory properties of cerium oxide nanoparticles. Small. 2009;5:2848-2856.

69. Singh S, Kumar A, Karakoti A, Seal S, Self WT. Unveiling the mechanism of uptake and sub-cellular distribution of cerium oxide nanoparticles. Mol Biosyst. 2010;6:1813-1820.

70. Zhou X, Wong LL, Karakoti AS, Seal S, McGinnis JF. Nanoceria inhibit the development and promote the regression of pathologic retinal neovascularization in the Vldlr knockout mouse. PLoS One. 2011;6:e16733.

71. Clark A, Zhu A, Sun K, Petty HR. Cerium oxide and platinum nanoparticles protect cells from oxidant-mediated apoptosis. J Nanopart Res. 2011;13:5547-5555.

72. Chen J, Patil S, Seal S, McGinnis JF. Rare earth nanoparticles prevent retinal degeneration induced by intracellular peroxides. Nat Nanotechnol. 2006;1:142-150.

73. Xia T, Kovochich M, Liong M, et al. Comparison of the mechanism of toxicity of zinc oxide and cerium oxide nanoparticles based on dissolution and oxidative stress properties. ACS Nano. 2008;2:2121-2134.

74. Lin W, Huang YW, Zhou XD, Ma Y. Toxicity of cerium oxide nanoparticles in human lung cancer cells. Int J Toxicol. 2006;25:451-457.
OncoTargets and Therapy

\section{Publish your work in this journal}

OncoTargets and Therapy is an international, peer-reviewed, open access journal focusing on the pathological basis of all cancers, potential targets for therapy and treatment protocols employed to improve the management of cancer patients. The journal also focuses on the impact of management programs and new therapeutic agents and protocols on

\section{Dovepress}

patient perspectives such as quality of life, adherence and satisfaction The manuscript management system is completely online and includes a very quick and fair peer-review system, which is all easy to use. Visit http://www.dovepress.com/testimonials.php to read real quotes from published authors. 